\title{
THE ENDOCANNABINOID SYSTEM AND CARDIOVASCULAR DISEASE
}

By Pal Pacher, MD, PhD, FAPS, FAHA

Laboratory of Physiologic Studies, National Institutes of Health/NIAAA, Bethesda, MD, USA pacher@mail.nih.gov

\section{Introduction}

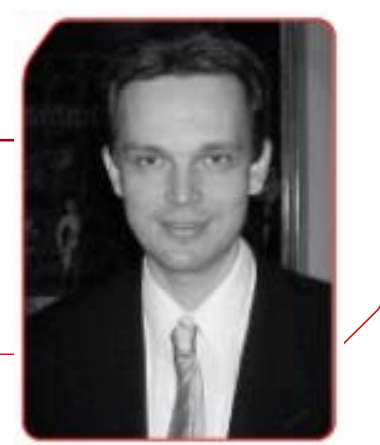

Cannabinoids and their endogenous and synthetic analogs exert complex cardiovascular effects both in vitro and in vivo mediated by cannabinoid receptor-dependent and -independent mechanisms. The cannabinoid $\mathrm{CB} 1$ and $\mathrm{CB} 2$ receptors and the endocannabinoid degrading enzymes are expressed in the myocardium [1-3], human coronary artery endothelial and smooth muscle cells $[4,5]$, and infiltrating inflammatory cells, among many other tissues/cells [6]. In experimental animals and in humans (depending on the route of administration, duration, and the dose) these cardiovascular effects may include CB1-mediated bradycardia/tachycardia, hypotension, and depressed cardiac contractility involving modulation of autonomic outflow through sites of action at presynaptic autonomic nerve terminals and in the central nervous system, as well as direct effects on myocardium and the vasculature [7]. In spite of the above mentioned cardiovascular effects of endocannabinoids, the endocannabinoid system (ECS) appears to play a limited role in cardiovascular regulation under normal physiological conditions. However, in various disease conditions, the ECS may become overactivated and play important protective and/or detrimental roles.

\section{Key Points}

- The cannabinoid CB1 and CB2 receptors and endocannabinoid degrading enzymes are present in cardiovascular tissues.

- Activation of cardiovascular CB1 receptors leads to hypotension and decreased cardiac contractility. However, the role of myocardial CB2 receptors is still elusive.

- The ECS plays a limited role in cardiovascular regulation under normal physiological conditions.

- In various forms of shock and heart failure, the ECS may become overactivated and contribute to depressed cardiovascular function, which can be prevented or attenuated by CB1 antagonists.

- The ECS may also be activated as a compensatory mechanism in various forms of hypertension to limit pathologically increased blood pressure and myocardial contractility.

- CB1 antagonists exert various cytoprotective and antiinflammatory effects in multiple unrelated preclinical disease models and also in patients with obesity and/or metabolic syndrome.

- Activation of CB2 receptors in inflammatory cells and endothelium attenuates TNF- $\alpha$-induced endothelial inflammatory response, chemotaxis, and adhesion of inflammatory cells to the activated endothelium, and consequent release of various proinflammatory mediators, which may underlie the beneficial effects of CB2 agonists in vascular inflammation, atherosclerosis, and myocardial ischemia/reperfusion injury. 


\section{State of the Art}

Activation of the ECS in inflammatory cells and cardiovascular tissues by bacterial endotoxin(s) has been implicated in cardiovascular collapse in various forms of shock (e.g., septic, hemorrhagic, and cardiogenic) and advanced liver cirrhosis (reviewed in [6]). In these conditions, treatment with CB1 antagonists prevented or reversed the hypotension and/or decreased myocardial contractility (reviewed in $[6,8])$. In rat models of acute and chronic myocardial infarction, studies with CB1 agonists/antagonists yielded conflicting results $[9,10]$. More recently, the role of the ECS was explored in a mouse model of doxorubicin(DOX)-induced heart failure [3]. Following doxorubicin administration, the tissue anandamide content, but not $\mathrm{CB} 1 / \mathrm{CB} 2$ receptor expression, was elevated in the myocardium and also in cardiomyocytes exposed to DOX in vitro, suggesting activation of the ECS. Pretreatment of mice with CB1 antagonists (rimonabant and AM281) not only improved DOXinduced cardiac dysfunction, but also attenuated the DOX-induced cell death both in vivo and in vitro. This cytoprotective effect suggests that the cardioprotective effect of $\mathrm{CB} 1$ antagonists in various cardiac pathologies may extend beyond beneficial hemodynamic effects. In fact, CB1 antagonists exert various anti-inflammatory and cytoprotective effects in multiple unrelated preclinical disease models [11-17]. Furthermore, rimonabant also attenuates multiple inflammatory markers [e.g., tumor necrosis factor- $\alpha$ (TNF- $\alpha$ ), C-reactive protein, etc.], plasma leptin and insulin levels, and increases plasma adiponectin in obese patients with metabolic syndrome and/or type 2 diabetes, thereby attenuating the development of cardiovascular risk factors associated with obesity/metabolic syndrome and diabetes [18-24]. On the basis of these studies, it was also suggested that rimonabant may have favourable effects in atherosclerosis. With this in mind, the results of the recent STRADIVARIUS clinical trial examining the effect of 18 months of rimonabant treatment on coronary disease progression in subjects with abdominal obesity/metabolic syndrome yielded somewhat disappointing results [19]. Rimonabant had no significant effect on the primary endpoint of coronary disease progression (the percent atheroma volume), however, it decreased the normalized total atheroma volume, which was the secondary endpoint [19]. The favourable effects of rimonabant on body weight and hormonal/metabolic parameters were similar to those observed in previous largescale trials.

Paradoxically, the ECS may also be activated as a compensatory mechanism in various forms of hypertension to limit pathologically increased blood pressure and myocardial contractility [6]. In this case, the enhancement of endogenous cannabinoid tone by inhibition of the anandamide degrading enzyme fatty acid amide hydrolase (FAAH) can decrease blood pressure and myocardial contractility [6].

The role of myocardial $\mathrm{CB} 2$ receptors during ischemia/reperfusion and other cardiovascular pathologies is still vague. In contrast, activation of CB2 receptors in inflammatory cells and endothelium attenuates TNF- $\alpha$-induced endothelial inflammatory response, chemotaxis, and adhesion of inflammatory cells to the activated endothelium, and consequent release of various proinflammatory mediators (key processes involved in the initiation and progression of atherosclerosis, restenosis, and reperfusion injury) $[8,25]$. Activation of CB2 receptors in human coronary smooth muscle cells 
decreases proliferation [5], which may have clinical implications for the treatment of atherosclerosis and restenosis.

\section{Priorities for Future Studies}

An increasing number of studies suggests that the beneficial effects of CB1 antagonists in various cardiomyopathies on contractile function may extend far beyond the simple inhibition of CB1mediated cardiovascular depressive effects of pathologically overproduced endocannabinoids in these disease conditions. Future studies using both knockout mice and additional selective CB1/2 agonists/antagonists must explore the possible interactions of the ECS with oxidative/nitrosative stress and related inflammatory pathways in models of myocardial ischemia/reperfusion, cardiomyopathies, heart failure, and atherosclerosis. Additional prospective studies should also examine if CB1 antagonist treatment leads to reduction of clinical events related to coronary disease. Novel therapeutic strategies targeting development of peripherally restricted CB1 antagonists may improve the benefit/risk ratio for this class of compounds by decreasing psychiatric side effects.

\section{References}

1. Batkai S, Pacher P, Osei-Hyiaman D, et al. Endocannabinoids acting at cannabinoid-1 receptors regulate cardiovascular function in hypertension. Circulation 2004; 110: 1996-2002.

2. Bonz A, Laser M, Kullmer S, et al. Cannabinoids acting on CB1 receptors decrease contractile performance in human atrial muscle. J Cardiovasc Pharmacol 2003; 41: 657-64.

3. Mukhopadhyay P, Batkai S, Rajesh M, et al. Pharmacological inhibition of CB1 cannabinoid receptor protects against doxorubicin-induced cardiotoxicity. J Am Coll Cardiol 2007; 50: 528-36.

4. Rajesh M, Mukhopadhyay P, Batkai S, et al. CB2-receptor stimulation attenuates TNF-alpha-induced human endothelial cell activation, transendothelial migration of monocytes, and monocyte-endothelial adhesion. Am J Physiol Heart Circ Physiol 2007; 293: H2210-8.

5. Rajesh M, Mukhopadhyay P, Hasko G, et al. CB2 cannabinoid receptor agonists attenuate TNF-alpha-induced human vascular smooth muscle cell proliferation and migration. Br J Pharmacol 2008; 153: 347-57.

6. Pacher P, Batkai S and Kunos G. The endocannabinoid system as an emerging target of pharmacotherapy. Pharmacol Rev 2006; 58: 389-462.

7. Pacher P, Batkai S and Kunos G. Cardiovascular pharmacology of cannabinoids. Handb Exp Pharmacol 2005; 599625.

8. Pacher $\mathrm{P}$ and Hasko G. Endocannabinoids and cannabinoid receptors in ischaemia-reperfusion injury and preconditioning. Br J Pharmacol 2008; 153: 252-62.

9. Wagner JA, Hu K, Bauersachs J, et al. Endogenous cannabinoids mediate hypotension after experimental myocardial infarction. J Am Coll Cardiol 2001; 38: 2048-54.

10. Wagner JA, Hu K, Karcher J, et al. CB(1) cannabinoid receptor antagonism promotes remodeling and cannabinoid treatment prevents endothelial dysfunction and hypotension in rats with myocardial infarction. Br $\mathrm{J}$ Pharmacol 2003; 138: 1251-8.

11. Berger C, Schmid PC, Schabitz WR, et al. Massive accumulation of N-acylethanolamines after stroke. Cell signalling in acute cerebral ischemia? J Neurochem 2004; 88: 1159-67.

12. Gary-Bobo M, Elachouri G, Gallas JF, et al. Rimonabant reduces obesity-associated hepatic steatosis and features of metabolic syndrome in obese Zucker fa/fa rats. Hepatology 2007; 46: 122-9. 
13. Kadoi Y and Goto F. Effects of AM281, a cannabinoid antagonist, on circulatory deterioration and cytokine production in an endotoxin shock model: comparison with norepinephrine. J Anesth 2006; 20: 284-9.

14. Malfitano AM, Laezza C, Pisanti S, et al. Rimonabant (SR141716) exerts anti-proliferative and immunomodulatory effects in human peripheral blood mononuclear cells. Br J Pharmacol 2008; 153: 1003-10.

15. Muthian S, Rademacher DJ, Roelke CT, et al. Anandamide content is increased and CB1 cannabinoid receptor blockade is protective during transient, focal cerebral ischemia. Neuroscience 2004; 129: 743-50.

16. Schafer A, Pfrang J, Neumuller J, et al. The cannabinoid receptor-1 antagonist rimonabant inhibits platelet activation and reduces pro-inflammatory chemokines and leukocytes in Zucker rats. Br J Pharmacol 2008; 154 : $1047-54$.

17. Sommer C, Schomacher M, Berger C, et al. Neuroprotective cannabinoid receptor antagonist SR141716A prevents downregulation of excitotoxic NMDA receptors in the ischemic penumbra. Acta Neuropathol 2006; 112: $277-86$.

18. Després JP, Golay A and Sjöström L. Effects of rimonabant on metabolic risk factors in overweight patients with dyslipidemia. N Engl J Med 2005; 353: 2121-34.

19. Nissen SE, Nicholls SJ, Wolski K, et al. Effect of rimonabant on progression of atherosclerosis in patients with abdominal obesity and coronary artery disease: the STRADIVARIUS randomized controlled trial. Jama 2008; 299: 1547-60.

20. Pi-Sunyer FX, Aronne LJ, Heshmati HM, et al. Effect of rimonabant, a cannabinoid-1 receptor blocker, on weight and cardiometabolic risk factors in overweight or obese patients: RIO-North America: a randomized controlled trial. JAMA 2006; 295: 761-75.

21. Ruilope LM, Després JP, Scheen A, et al. Effect of rimonabant on blood pressure in overweight/obese patients with/without co-morbidities: analysis of pooled RIO study results. J Hypertens 2008; 26: 357-67.

22. Scheen AJ, Finer N, Hollander P, et al. Efficacy and tolerability of rimonabant in overweight or obese patients with type 2 diabetes: a randomised controlled study. Lancet 2006; 368: 1660-72.

23. Van Gaal L, Pi-Sunyer X, Després JP, et al. Efficacy and safety of rimonabant for improvement of multiple cardiometabolic risk factors in overweight/obese patients: pooled 1-year data from the Rimonabant in Obesity (RIO) program. Diabetes Care 2008; 31 Suppl 2: S229-40.

24. Van Gaal LF, Rissanen AM, Scheen AJ, et al. Effects of the cannabinoid-1 receptor blocker rimonabant on weight reduction and cardiovascular risk factors in overweight patients: 1-year experience from the RIO-Europe study. Lancet 2005; 365: 1389-97.

25. Mach F, Montecucco F and Steffens S. Cannabinoid receptors in acute and chronic complications of atherosclerosis. Br J Pharmacol 2008; 153: 290-8. 